\title{
Chip to city: the future of mobility
}

\section{Stefan Jockusch}

Stefan Jockusch, "Chip to city: the future of mobility," Proc. SPIE 11148, Photomask Technology 2019, 1114802 (26 September 2019); doi: 10.1117/12.2540662

SPIE Event: SPIE Photomask Technology + EUV Lithography, 2019, Monterey, California, United States 


\title{
Chip to city: the future of mobility
}

\author{
Stefan Jockusch \\ Siemens Digital Industries Software, 5800 Granite Parkway Suite 600, Plano, TX 75024
}

\begin{abstract}
Cities of the future will be founded on the transmission of data between various intelligent systems, including the design, manufacturing, and deployment of autonomous vehicles. As automotive companies transition from building and selling cars to offering mobility services, integrated solutions for information technology, product lifecycle management, and various engineering domains will be critical to success. Among these engineering domains, the development of advanced system-on-chip (SoC) devices will be especially crucial to the success of autonomous vehicles. Autonomous vehicles demand bespoke SoC devices that are optimized for the specific challenges of automated driving. Legacy automotive manufacturers, automotive suppliers, and new entrants developing autonomous vehicles will need to adopt a crossdomain approach to semiconductor development that enables early verification and validation of SoCs within the context of an autonomous vehicle. Integrating such a portfolio into a digital automotive enterprise will enable companies as they strive to realize the potential of new mobility technologies.
\end{abstract}

Keywords: Autonomous drive, digital twin, SoC, semiconductor, high-level synthesis, emulation, functional safety, physical verification

\section{INTRODUCTION}

What will it require to build the cities of the future? In many respects, the cities of the future will be founded on common ground with cities of the past. Cities need access to resources, transportation systems to move people and goods, and various forms of infrastructure to support these systems. In the past, rivers were the linchpin of urban growth and prosperity. Rivers facilitated trading and commerce, cultural exchange, and transportation (Figure 1).

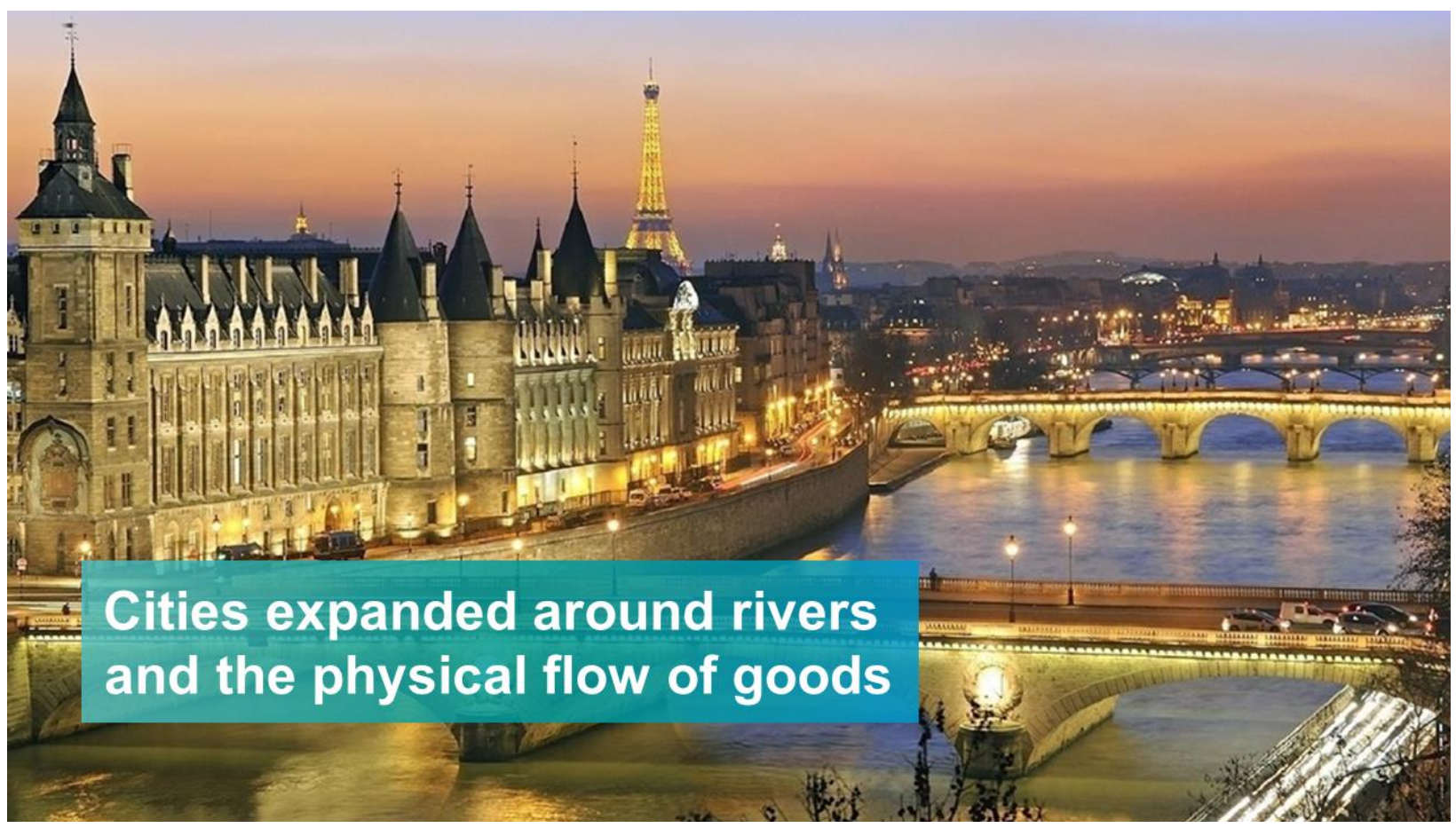

Photomask Technology 2019, edited by Jed H. Rankin, Moshe E. Preil, Proc. of SPIE Vol. 11148, $1114802 \cdot$ @ 2019 SPIE · CCC code: 0277-786X/19/\$21 · doi: 10.1117/12.2540662 
Figure 1: In the past, cities flourished along rivers as they provided trade, cultural exchange, and transportation.

Cities of the future will also prosper along rivers, but they will not be physical bodies of water. Future cities will grow upon digital "rivers", harnessing the flow of data to connect and transform everything. The proliferation of smart sensing devices, cloud computing, and faster network connections will blend the physical and digital worlds, affecting how we interact, consume goods, and move around.

The automotive industry is expected to shift from a model of building and selling cars towards providing mobility services through autonomous vehicles, micro-mobility, and integrated transportation networks. This change is expected to generate 1.5 trillion dollars in recurring revenues. Taking advantage of this opportunity requires a transformation in how we design, manufacture and deploy the vehicles of the future.

Digitalization is an inseparable combination of technology innovation and process innovation. Integrated solutions for information technology, product lifecycle management, and various engineering domains such as simulation, electrical systems, semiconductor development, and manufacturing, will be critical to success. Additionally, cloud-based analytics will feed data back into fleet management systems, manufacturing sites, and product development teams to create a closed loop of data continuity throughout the flow (Figure 2).

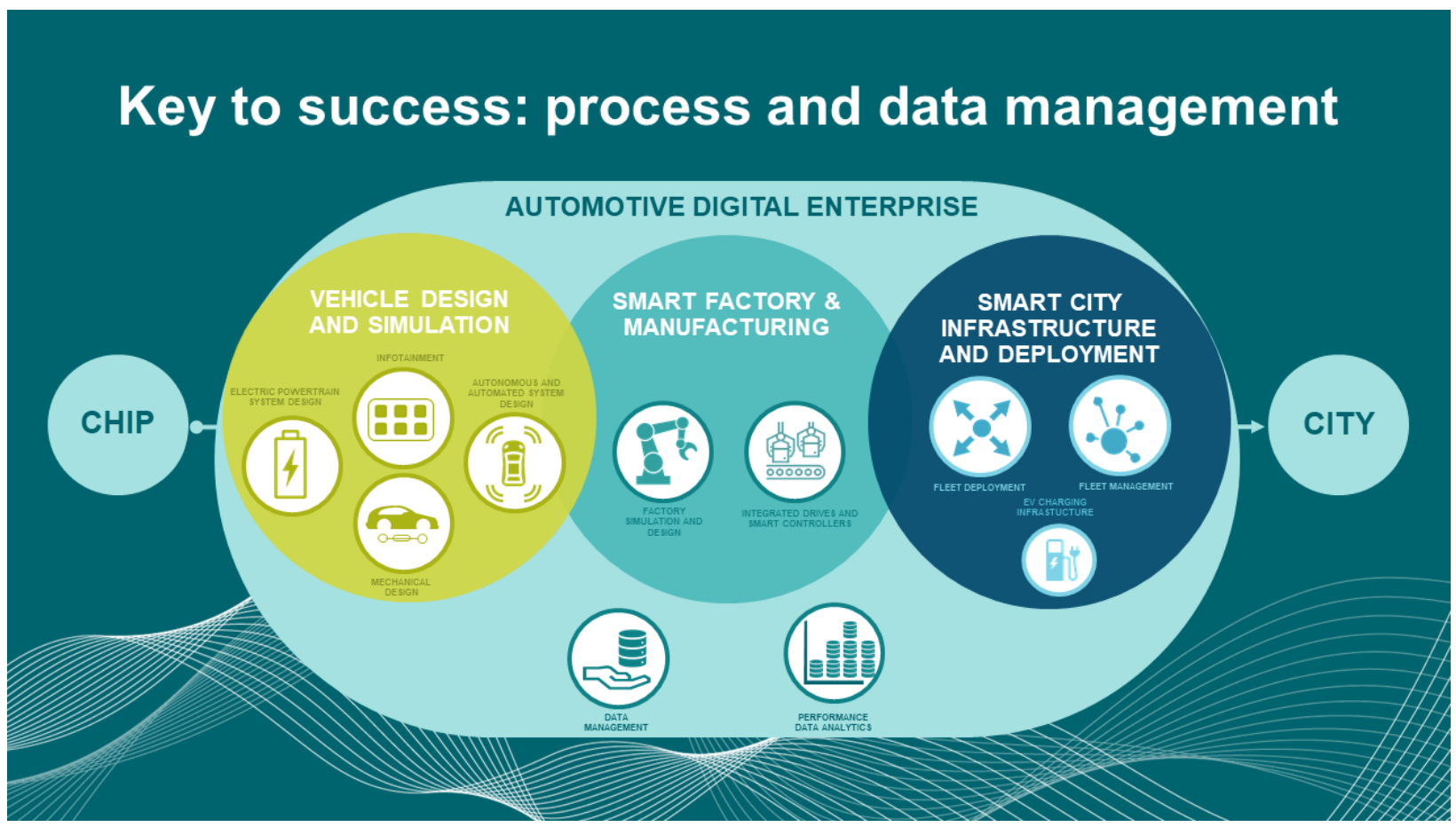

Figure 2: An automotive digital enterprise features integrated solutions across domains and applications, with a closed-loop back to design and manufacturing.

Leading companies are already investing in their digitalization strategy. This early investment will create an operational advantage amounting to a 10-20\% reduction in total operational cost. With no digitalization strategy, businesses continue the practice of relying on intuition to make decisions during product development, manufacturing, and deployment, ultimately risking failure in the not-so-distant future. It is critical for companies to prepare for tomorrow by creating operational advantage through the improvement of productivity with today's fleet in advance of the future mobility opportunities.

By embracing digitalization, automotive manufacturers can create digital streams of their own, connecting engineering domains such as electrical, mechanical, manufacturing, and software. While individual vehicle complexity continues to grow - whether measured in lines of code, or the number of functions, available vehicle variants, or launches per year - 
the real complexity in automotive comes from the increasing need to collaborate across domains, applications, and the ecosystem. A comprehensive strategy to digitalize the design, production, deployment, and monitoring of vehicles must remove the boundaries that have existed, empowering automotive companies to create autonomous and connected mobility systems.

Autonomous vehicle systems demand an integrated, cross-domain design approach. Automotive companies are attempting to integrate robust sensor suites and bleeding-edge system-on-chip (SoC) devices into vehicle architectures that are already extremely sophisticated. The SoCs that will control the vehicle are of particular importance. These chips must be purpose-built for the task of automated driving, and verified not just for functionality and reliability, but in real driving scenarios as part of a physical or virtual vehicle prototype.

\section{AT THE CENTER OF THE COMPLEXITY CHALLENGE: SOC DEVELOPMENT FOR AUTONOMOUS DRIVE}

Autonomous vehicle architectures are converging, with larger and more powerful domain-controller SoCs connected to a centralized processing unit, all implementing artificial intelligence functions. To date, many autonomous vehicle programs have used CPUs and GPUs, designed for data center or personal computing applications, as these domain controllers and central processors. These solutions worked well for early testing and development tasks, but are not equipped to meet the power, performance, and space requirements of a true autonomous vehicle SoC. Chip designers are discovering that meeting these requirements will require new silicon and system architectures, developed around artificial intelligence, machine learning, and high-volume information processing.

Developing bespoke SoCs to meet these exacting requirements is one of the most difficult, and critical challenges that autonomous vehicle programs must overcome to achieve commercial success. The ability of these chips to navigate a vehicle safely and reliably through a real-world environment will be a key differentiator in the autonomous vehicle market. The safest and most reliable system will garner the greatest public trust, and thus the most favor in the marketplace.

A portfolio of advanced integrated circuit (IC) design and verification solutions will be a critical piece of the digital automotive enterprise. Advanced IC design and verification solutions can help companies realize their SoC designs, verify them, maximize their post-manufacturing yield, and ensure their reliability over long lifetimes. These solutions are also critical to an overall digitalization strategy. Integrations between SoC development and vehicle program development will enable the early verification and validation of AV chips in the context of simulated vehicle subsystems, software, and even detailed city environments. This integration will result in safe and reliable SoC designs that are optimized for the specific vehicle platform in which they will operate.

\subsection{The race against the clock: shortening automotive IC design cycles}

To meet the high-performance and low-power requirements of autonomous vehicles, SoC designers will need to create bespoke silicon architectures optimized for artificial intelligence algorithms. Using traditional design methodologies will take far too long, as the complexity of designs increases and verification time rises. To lower the investment in time and labor, many companies are looking for a proven solution that can increase their productivity and design quality, while accelerating their time-to-market. This explains the increasing interest in high-level synthesis (HLS).

HLS takes high-level descriptions of the design functionality in SystemC or C++ and synthesizes them into RTL. Designing at a higher level of abstraction accelerates the completion of initial designs by separating the specification of the chip functionality from the implementation (Figure 3). Designers must only describe what the chip needs to do, without delving into how it accomplishes such functionality. Then, the HLS tool automatically generates RTL to implement the described functionality. 


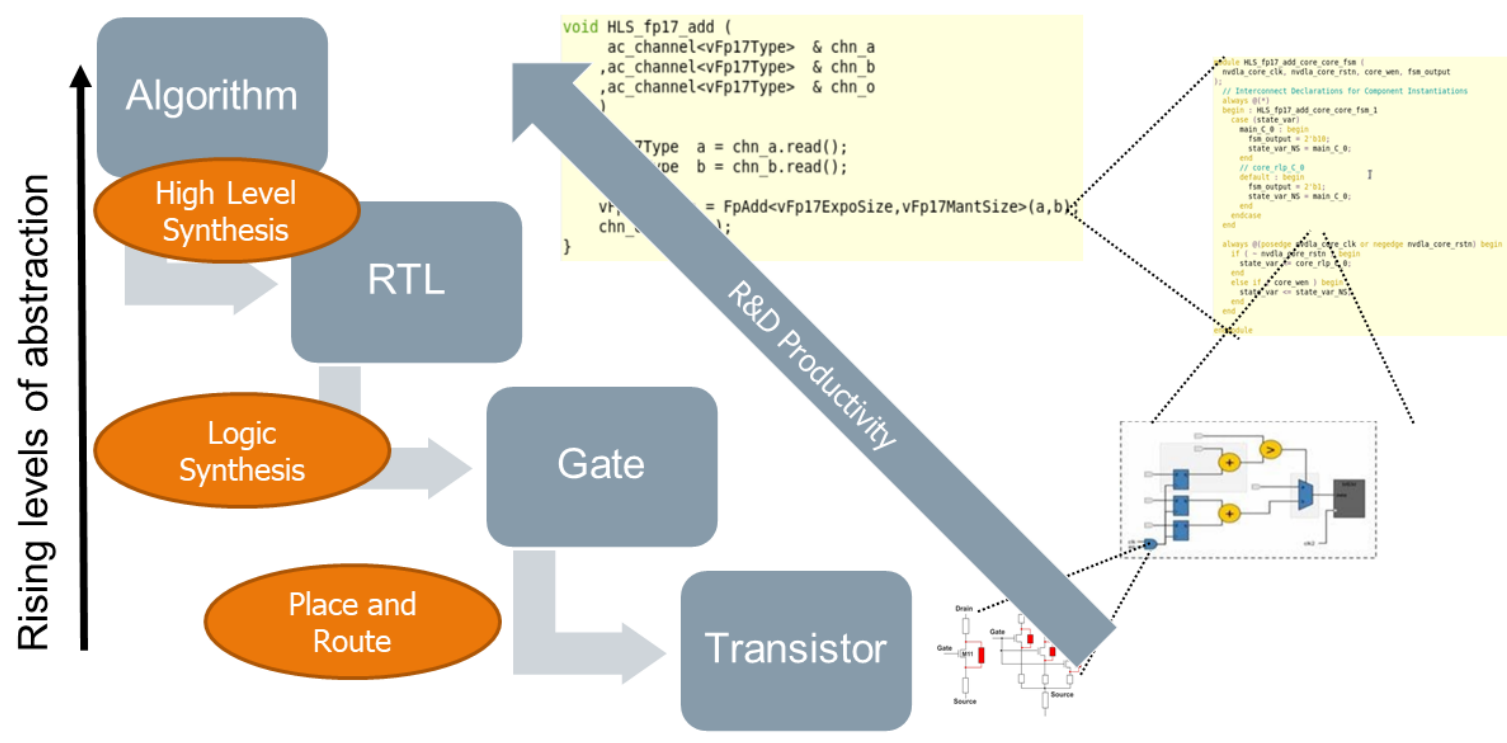

Figure 3: HLS rises the design abstraction level to improve design productivity.

Using HLS to design at a higher level of abstraction can reduce design times to a few months, and requires half as much code as a traditional RTL flow. Late functional changes, new features, or even a migration between technology nodes or from FPGA to ASIC can be incorporated without impacting the design schedule. HLS also enables the design team to explore hundreds of design variants to optimize the power, performance, and area of the chip. Design space exploration results in higher quality designs compared to hand-coded RTL.

\subsection{Functional safety, verification \& design for safety}

Despite the numerous challenges involved in designing SoCs for AVs, the most substantial obstacle to the success of $\mathrm{AVs}$ is earning the trust of the public. One way that AV manufacturers can establish this trust is to demonstrate the safety and reliability their platform through safety standards and certification. To that end, the automotive industry has established a set of procedures and standards focused on the safety of electrical and electronic systems, known as functional safety. Functional safety is the reduction of the risk of electrical and electronic components malfunctioning due to failures. In the automotive industry, these procedures and requirements have been formalized in the ISO 26262 standard. ISO 26262 requires that electronics be tested for random hardware failures and systematic faults (Figure 4). 


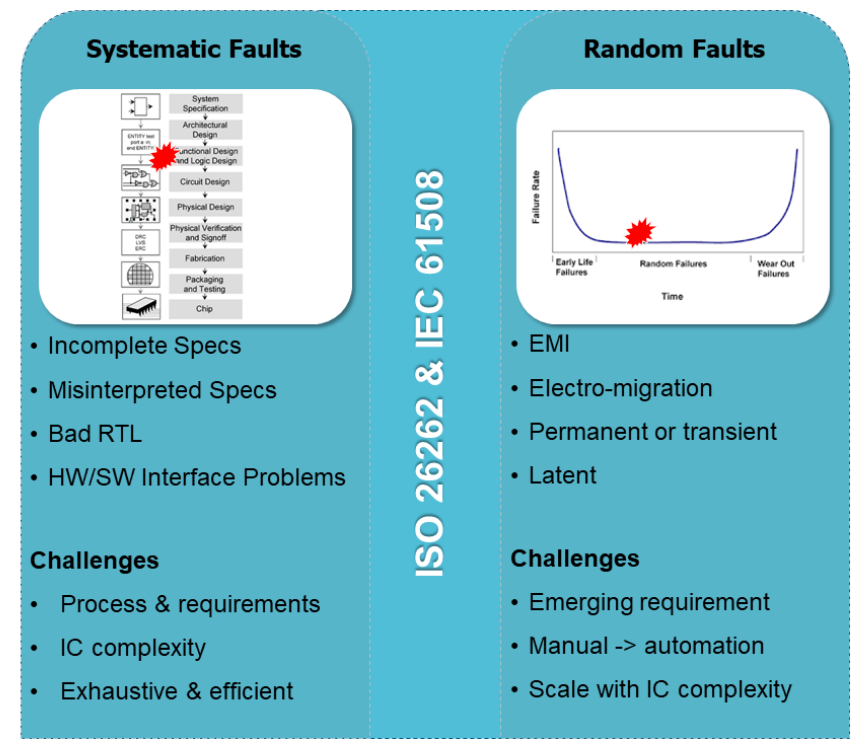

Figure 4: ISO 26262 requires that electronics are tested for systematic and random faults.

Systematic faults are those that prevent an integrated circuit from operating correctly according to the product specifications. These could be design bugs, hardware/software interface problems, misinterpreted or incomplete specifications and so forth. The goal is to eliminate systematic faults through robust design procedures, qualified EDA tools, and formal requirements.

On the other hand, random hardware faults occur over time as the IC operates. Random hardware faults can be caused by electromagnetic interference (EMI), electro-migration, and other electrical phenomena. Some of these faults are transient, and will disappear with time, while others are permanent. In either case, a random hardware failure in a mission-critical autonomous IC has potentially catastrophic consequences. Therefore, ISO 26262 requires that chips continue to operate, or fail safely, in the event of a random hardware fault.

Verifying the functional safety of an automotive SoC involves the analysis of a design to identify potential failure modes, the insertion of safety mechanisms to mitigate failures, and verification to demonstrate the effectiveness of inserted safety mechanisms. Verifying the SoC in isolation, however, is not enough to guarantee its safe operation once inside the vehicle. For that, the chip must be tested in real operating conditions. It is not feasible to test every possible safety scenario in the real world. As vehicles progress from level 1 to level 5 automation, the number of potential scenarios that must be investigated to properly validate a vehicle explodes into the millions. As a result, estimates predict that it will require more than eight billion miles to fully test and verify the safety and functionality of an autonomous car. The only way to achieve this amount of verification is with a virtual testing environment employed early in the design process.

Hardware emulation supports model, software, and hardware in-the-loop verification. It provides an environment to test, program, and debug an IC or an entire autonomous vehicle platform before any chip or vehicle hardware is available. This testing environment merges three data types (Figure 5). First is sensor data. To generate this data without sensor hardware, advanced physics-based sensor simulations feed the hardware emulation with simulated LiDAR, radar, camera, and other sensor data. Second is compute data that is provided by the emulator running the autonomous drive IC. Third, a mechatronic simulator provides actuator data from steering, braking, and drivetrain systems. Advanced sensor simulators can also generate traffic patterns and simulate V2X communications to fully test the capabilities of an autonomous vehicle platform. 


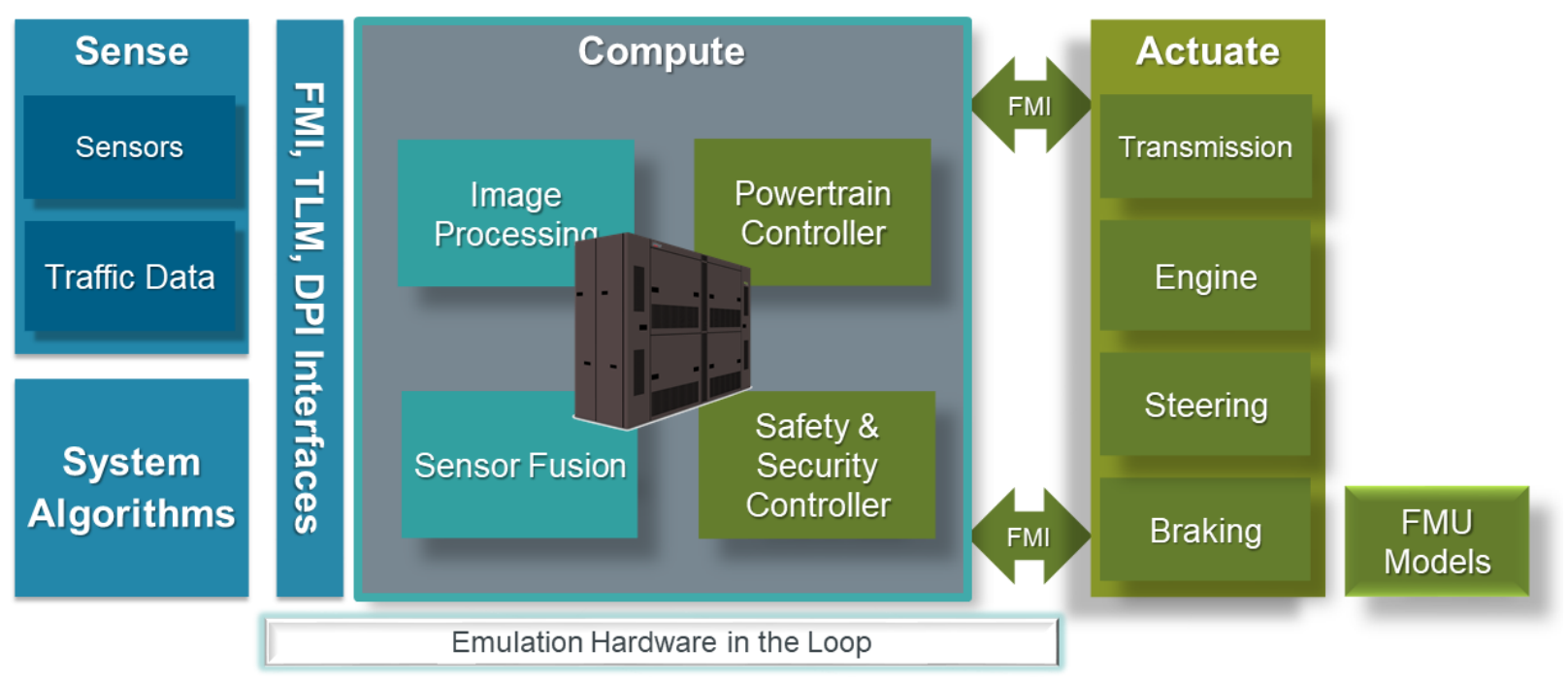

Figure 5: Hardware emulation can fuse sensor, compute, and actuation data to create a testing environment for autonomous vehicle platforms.

\subsection{Physical reliability verification}

Now, the SoC design team has optimized the chip through rapid iteration enabled by HLS, verified for its functionality and functional safety, and even tested it in a realistic virtual driving environment with simulated sensor and mechatronic data. Next, the physical reliability of the design must be verified to ensure that the design will survive the manufacturing process with sufficient yield, and will operate as intended in the real-world. The hand-off from design to manufacturing is a critical juncture in the IC design process; it is where the rubber meets the road.

Understanding the reliability needs of autonomous vehicle ICs can be challenging, particularly for companies just entering this market. Typical automotive ICs are expected to function for up to thirty years in temperatures that can range from $-40^{\circ} \mathrm{C}$ to $150^{\circ} \mathrm{C}$, and electrical loads into the hundreds of volts. The harsh driving environment that automotive electronics must operate in, combined with the high reliability requirements for verification of these ICs, creates design and verification challenges that are not commonly encountered when developing ICs used in less demanding settings. Design standards will mandate acceptable criteria for electrostatic discharge (ESD) or electrical overstress (EOS) compliance, but they cannot articulate the design trade-offs or best practices needed to meet such criteria.

The traditional IC physical verification tools of design rule checking (DRC), layout vs. schematic (LVS) comparison, and electrical rule checking (ERC) can efficiently identify and solve very specific layout and circuitry issues within your IC design. But they cannot understand the holistic impact of device implementation in the context of a larger circuit. Standard physical verification tools struggle to consider the net connectivity and the physical layout of a device in the same framework.

Fortunately, a new class of IC reliability verification tool is able to consider these problematic realms in a cohesive environment. Created out of the need to improve the coverage of IC reliability verification in a circuit aware context, these tools allow focused analysis on how circuits are implemented from both a circuit topology and layout perspective. As part of this analysis, external constraints can be leveraged to direct the intent of checks and help determine which 
circuits are out of compliance. A reliability verification tool that can understand and assess those constraints is essential to identifying reliability issues and ensuring compliance with reliability requirements and industry standards.

One common example is protection and verification against time-dependent dielectric breakdown (TDDB) in interconnects (often called voltage-aware DRC), where reliability verification in electrical overstress (EOS) environments plays a critical role. This issue requires larger design areas to avoid failure, but is critical to mitigate in high reliability IC designs.

Next, designers must optimize the physical layout of the chip for successful manufacturing. Design for manufacturing (DFM) solutions can help designers by automatically performing layout optimizations, simulating manufacturing processes, or managing lithographic hotspots before tape-out. DFM solutions automatically measure changes in yield that result from proposed layout modifications. This gives designers the ability to select layout modifications that will maximize manufacturing yield and reliability of the chips.

\section{SOC DESIGN AND VERIFICATION AS PART OF THE DIGITAL AUTOMOTIVE ENTERPRISE}

The success of autonomous vehicles hinges on the ability of a system of advanced sensors and powerful chips to perceive and process an immense amount of data in real-time. As a result, these chips will require never-before-seen architectures to meet the power, performance, and area required for autonomous drive. Furthermore, autonomous ICs will need to function with near flawless reliability and accuracy, despite harsh environmental conditions, for extended periods of time, much longer than ICs in traditional consumer electronics.

As automotive startups, established OEMs, and systems companies vie to be the first to market, they will need a portfolio of advanced design automation and lifecycle management tools. Siemens PLM and Mentor are uniquely positioned to provide such tools, with leading solutions in HLS, functional safety and verification, emulation, physical reliability verification, and an industry-first full-vehicle validation platform.

Integrating such a portfolio into a digital automotive enterprise will enable companies as they strive to realize the potential of new mobility technologies. As boundaries between engineering domains are removed, companies will gain a holistic perspective on their vehicle programs, enabling unprecedented improvements in technology and processes. Such comprehensive digitalization throughout an organization, leveraging solutions from chip to city, will lay the foundation for automotive OEMs, startups, and mobility providers as they help construct the cities of tomorrow. 